\title{
Large Size LSO and LYSO Crystals for Future High Energy Physics Experiments
}

\author{
Jianming Chen, Member, IEEE, Rihua Mao, Member, IEEE, Liyuan Zhang, Member, IEEE, and \\ Ren-Yuan Zhu, Senior Member, IEEE
}

\begin{abstract}
Following our previous studies, a further investigation on three long $\left(2.5 \times 2.5 \times 20 \mathrm{~cm}^{3}\right)$ LSO and LYSO crystal samples was carried out, and was compared to a long BGO sample of the same size. The optical and scintillation properties, including transmittance, photo-luminescence and excitation spectra, were measured. The result of light output and light response uniformity, measured by using both PMT and APD, are reported. Their applications in future high energy physics experiments are discussed.
\end{abstract}

Index Terms-Crystal, light output, lutetium oxyorthosilicate, lutetium yttrium oxyorthosilicate, photo luminescence, radiation damage, scintillator, transmission.

\section{INTRODUCTION}

$\mathbf{I}$ $\mathrm{N}$ the last decade, mass production capabilities of the Gadolinium Orthosilicate $\left(\mathrm{Gd}_{2} \mathrm{SiO}_{5}, \mathrm{GSO}\right)$ [1], lutetium oxyorthosilicate $\left(\mathrm{Lu}_{2} \mathrm{SiO}_{5}, \mathrm{LSO}\right)$ [2] and lutetium-yttrium oxyorthosilicate $\left(\mathrm{Lu}_{2(1-x)} \mathrm{Y}_{2 x} \mathrm{SiO}_{5}\right.$, LYSO) [3], [4] crystals have been established for the medical industries. Because of their high stopping power and fast bright scintillation, LSO and LYSO crystal scintillators have also attracted broad interest in the high energy physics community for future experiments, such as a super B factory [5] and the international linear collider (ILC) [6]. Following our previous studies on two long LYSO samples [7] this report presents a further study on large size LSO and LYSO samples. In addition to the light output measured by using vacuum photomultiplier tubes (PMT), solid state silicon avalanche photodiodes (APD) are also used as readout device for crystal's light output and light response uniformity.

Fig. 1 shows typical quantum efficiencies of a PMT with bi-alkali cathode (Hamamatsu R2059 PMT) and an APD (Hamamatsu S8664), as well as the photo luminescence spectra of LSO/LYSO, BGO and CsI(Tl) crystals. Table I summarizes the numerical values of the photo luminescence weighted average quantum efficiencies for various readout devices used in this study, which can be used to convert the measured photoelectron numbers to the absolute light output in photon numbers.

Fig. 2 is a photo showing four long crystal samples with dimensions of $2.5 \times 2.5 \times 20 \mathrm{~cm}$. They are, from top to bottom:

Manuscript received August 17, 2006; revised February 8, 2007. This work was supported in part by the U.S. Department of Energy under Grant DE-FG03-92-ER40701.

The authors are with the California Institute of Technology, Pasadena, CA 91125 USA (e-mail: zhu@hep.caltech.edu).

Color versions of one or more of the figures in this paper are available online at http://ieeexplore.ieee.org.

Digital Object Identifier 10.1109/TNS.2007.897823

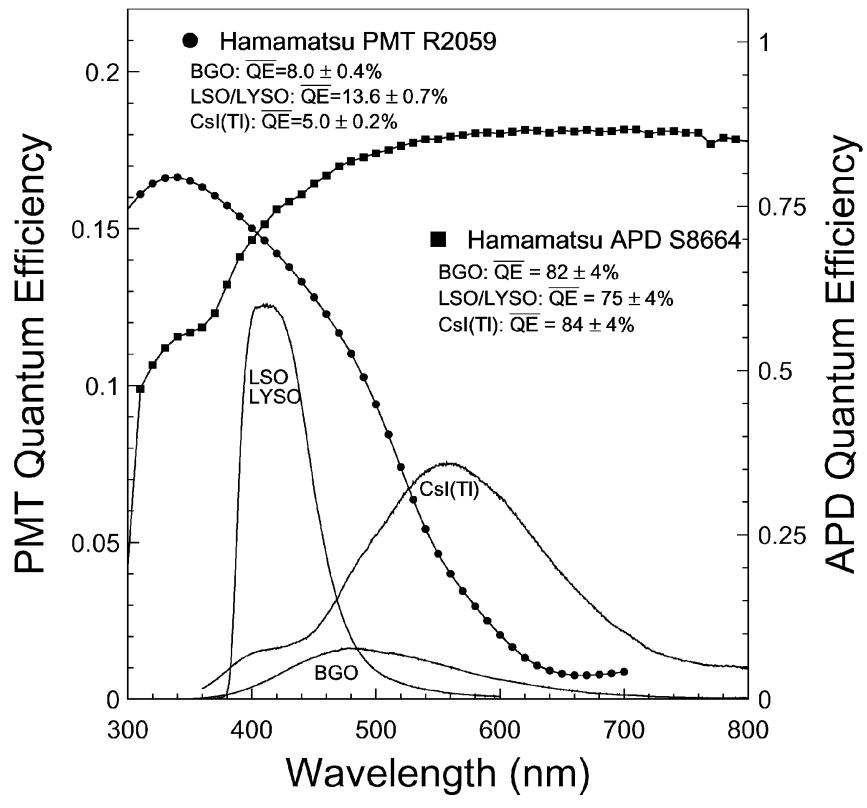

Fig. 1. The quantum efficiencies of a Hamamatsu R2059 PMT (solid dots) and a Hamamatsu S8664 APD (solid squares) are shown as a function of wavelength together with the photo luminescence spectra of the LSO/LYSO, BGO and $\mathrm{CsI}(\mathrm{Tl})$ samples, where the area under the photo luminescence curves is roughly proportional to corresponding absolute light output.

a BGO sample from Shanghai Institute of Ceramics (SIC), two LYSO samples from Crystal Photonics, Inc. (CPI) and SaintGobain Ceramics \& Plastics, Inc. (Saint-Gobain) and a LSO sample from CTI Molecular Imaging (CTI). It is noted that the CPI long LYSO sample has chips at the corners and surfaces since CPI does not have adequate polishing and treatment facilities for such large size samples [8]. Also shown in Fig. 2 are two small cubic samples each with dimension of 1.5 radiation length $(1.7 \times 1.7 \times 1.7 \mathrm{~cm})$ from these four vendors. These long LSO and LYSO samples were specially prepared by manufactures for the authors. Results for LYSO crystal samples of such size with PMT readout were first reported in our previous publication [7]. Results for LSO and LYSO crystal samples of such size with APD readout are first reported in this paper.

According to the manufacturers, the yttrium content is about $5 \%$ for the CPI LYSO [8] and about $10 \%$ for the Saint-Gobain LYSO [9]. The nominal cerium doping level is $0.2 \%$ for the CTI LSO [10] and the CPI LYSO [8], and is less than $1 \%$ for the Saint-Gobain LYSO [9]. The actual cerium concentration in these crystals, however, would be less than the nominal value and its distribution along the long sample's axis also varies because of the the cerium segregation process during crystal 
TABLE I

Photo Luminescence Weighted QuANTUM EFFICIENCIES (\%)

\begin{tabular}{cccc}
\hline \hline Photo Luminescence & LSO/LYSO & BGO & CsI(Tl) \\
\hline Hamamatsu R1306 PMT & $12.9 \pm 0.6$ & $8.0 \pm 0.4$ & $5.0 \pm 0.3$ \\
Hamamatsu R2059 PMT & $13.6 \pm 0.7$ & $8.0 \pm 0.4$ & $5.0 \pm 0.3$ \\
Photonis XP2254b & $7.2 \pm 0.4$ & $4.7 \pm 0.2$ & $3.5 \pm 0.2$ \\
\hline Hamamatsu S2744 PD & $59 \pm 4$ & $75 \pm 4$ & $80 \pm 4$ \\
Hamamatsu S8664 APD & $75 \pm 4$ & $82 \pm 4$ & $84 \pm 4$ \\
\hline \hline
\end{tabular}

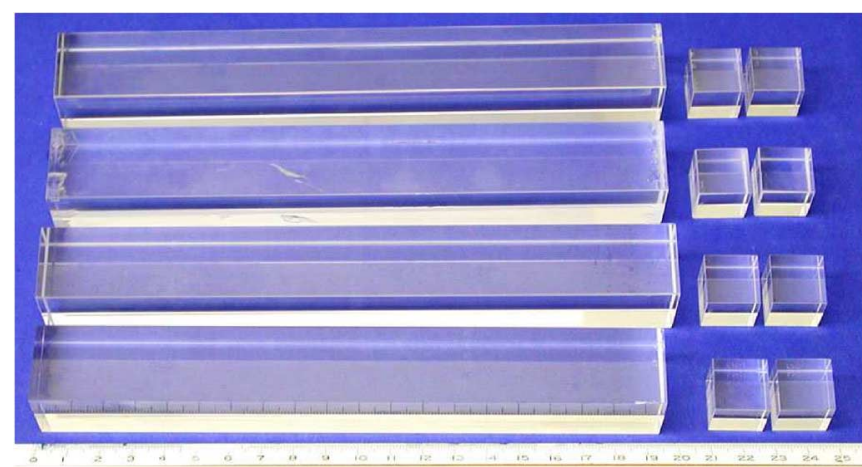

Fig. 2. A photo showing four long $(2.5 \times 2.5 \times 20 \mathrm{~cm})$ crystal samples, from top to bottom: SIC BGO, CPI LYSO, SG LYSO and CTI LSO, together with two corresponding small cubic samples $\left(1.5 \mathrm{X}_{0}\right.$ cube) from each manufacturer.

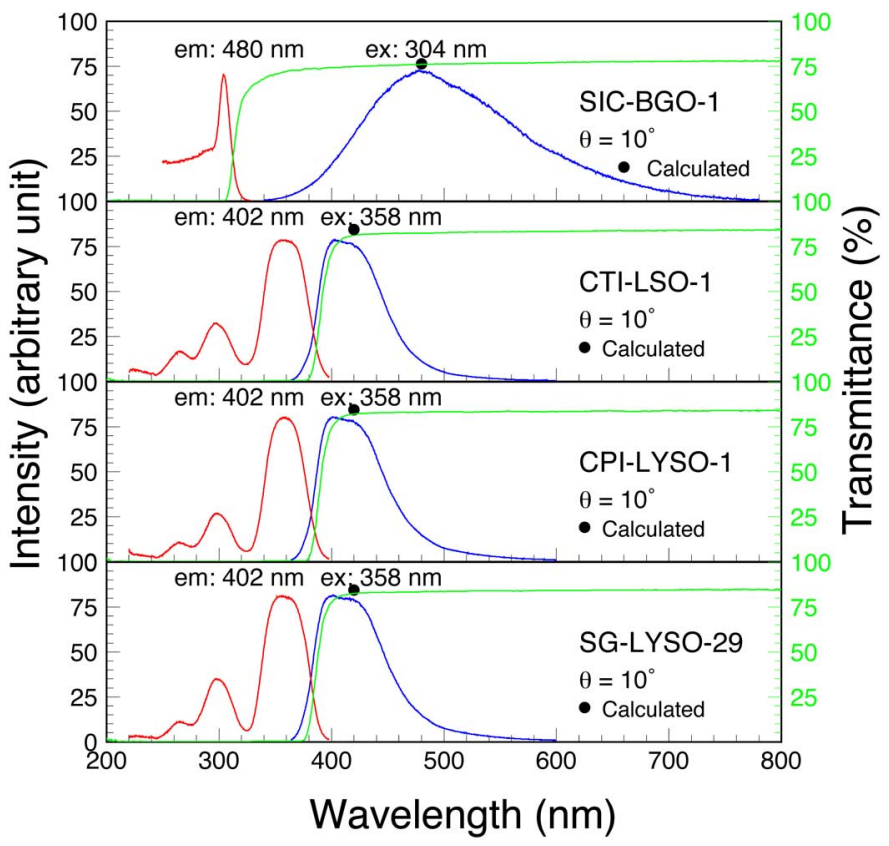

Fig. 3. The excitation and photo luminescence spectra (left scale) and the longitudinal transmittance spectra (right scale) are shown as a function of wavelength for four cubic samples.

growth. The details of crystal manufacturing, such as the seed or tail in growth, however, are not provided by the manufacturers. All surfaces of these samples are polished. Unless specified, no thermal treatment was applied before initial measurements.

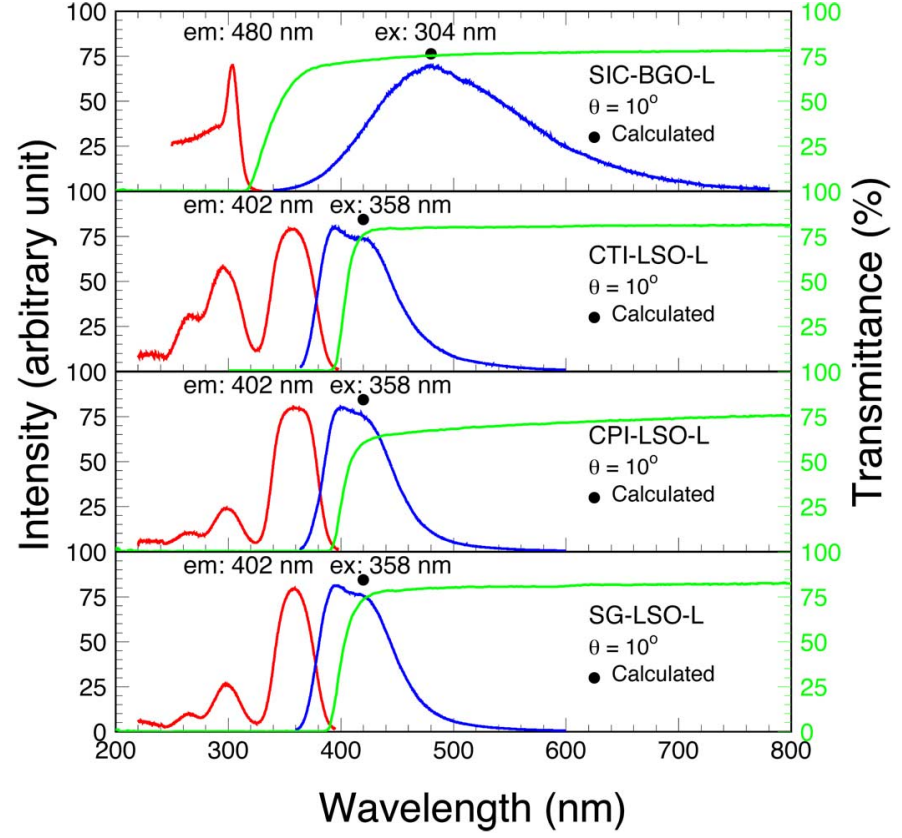

Fig. 4. The excitation and photo luminescence spectra (left scale) and the longitudinal transmittance spectra (right scale) are shown as a function of wavelength for four long samples.

\section{SCintillation ANd Optical Properties}

The transmittance spectra were measured by using a Hitachi U-3210 UV/visible spectrophotometer with double beam, double monochromator and a large sample compartment equipped with a custom Halon coated integrating sphere. The systematic uncertainty in repeated measurements is about $0.3 \%$. Taking into account multiple bouncing between two end surfaces, the theoretical limit of transmittance without internal absorption, $T_{s}$, can be calculated as [11]

$$
T_{s}=(1-R)^{2}+R^{2}(1-R)^{2}+\cdots=(1-R) /(1+R),
$$

where

$$
R=\frac{\left(n_{\text {crystal }}-n_{\text {air }}\right)^{2}}{\left(n_{\text {crystal }}+n_{\text {air }}\right)^{2}} .
$$

A comparison of the measured transmittance and $T_{s}$ may reveal internal absorption. 


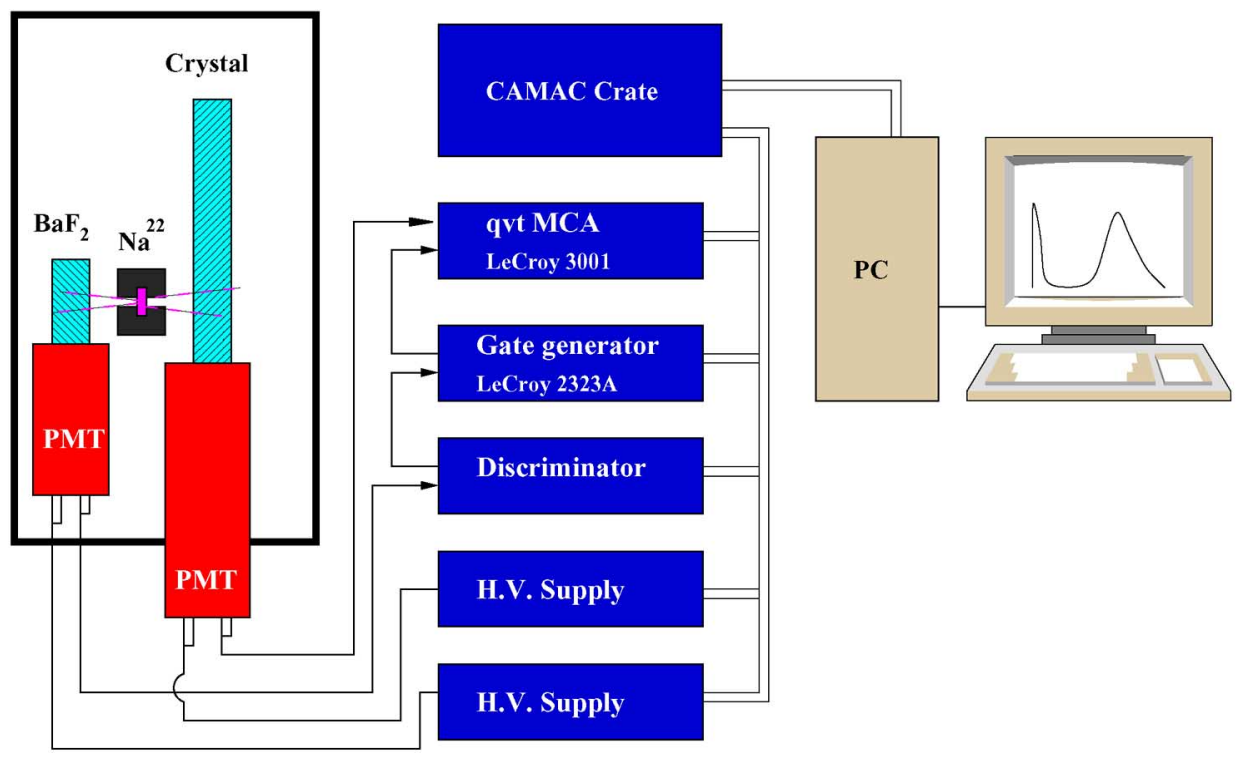

Fig. 5. A schematic showing the set-up used for the light output measurement with the PMT readout and coincidence trigger for long samples.

The excitation and photo luminescence spectra were measured by using a Hitachi F-4500 fluorescence spectrophotometer. UV excitation light was directed onto a bare surface of the sample, and the crystal samples were oriented so that its photo luminescence light is not affected by sample's internal absorption.

Figs. 3 and 4 show a comparison of the transmittance, photo luminescence and excitation spectra as a function of wavelength for four cubic and long samples respectively. The solid black dots in these plots show the theoretical limit of the transmittance at the photo luminescence peak, calculated by using (1). All cubic samples have their transmittance approaching the theoretical limits, indicating small internal absorption. The longitudinal transmittance of long samples, however, are lower than the theoretical limits, indicating cumulated internal absorption.

It is interesting to note that the UV absorption edge in the longitudinal transmittance spectra of the LSO and LYSO long samples cuts into the photo luminescence spectra and thus affects crystal's light output, which is not the case for the long BGO sample. As discussed in our previous work [7], this indicates that the light output of the LSO and LYSO long samples is partially self-absorbed. Alternatively, an improvement of the transmittance in the direction toward a shorter wavelength, e.g., via appropriate doping or optimizing Ce concentration etc., would effectively improve the light output for LSO and LYSO crystals. This kind of improvement was achieved in previous crystal development programs for high energy physics.

\section{Result With PMT Readout}

A Hamamatsu R1306 PMT with bialkali photo cathode was used to measure crystal's energy resolution for $\gamma$-rays from radioactive sources. The scintillation light decay kinetics was measured using a Photonis XP2254b PMT, which has a multi-alkali photo cathode and a quartz window. In all these measurements one end of the sample was coupled to the PMT with Dow Corning 200 fluid, while all other faces of the sample were wrapped with Tyvek paper. A collimated ${ }^{137} \mathrm{Cs}$ source

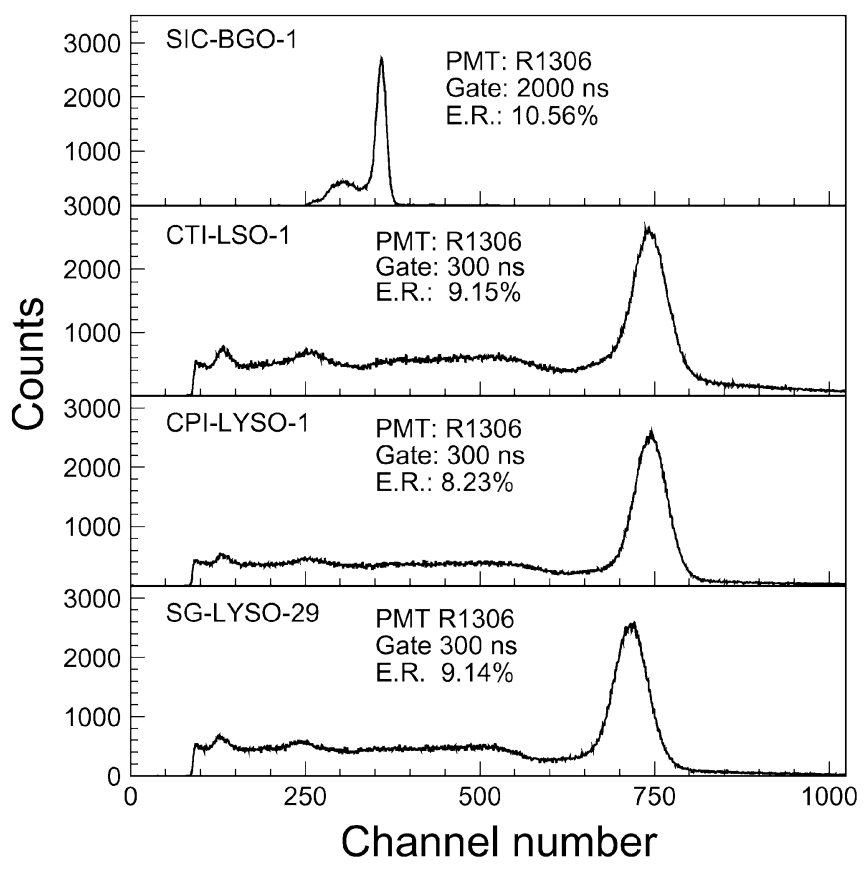

Fig. 6. The spectra of $0.662 \mathrm{MeV} \gamma$-rays from a ${ }^{137} \mathrm{Cs}$ source measured by using the R1306 PMT are shown for four cubic samples.

was used to excite the small samples. To reduce the effect of intrinsic natural radioactivity, a collimated ${ }^{22} \mathrm{Na}$ source was used for long samples with a coincidence trigger provided by a $\mathrm{BaF}_{2}$ crystal, as shown in Fig. 5. In both measurements, the $\gamma$-ray peak position was determined by a simple Gaussian fit.

Figs. 6 and 7 show $\gamma$-ray energy spectra measured for the cubic and long samples respectively, by using the R1306 PMT. The FWHM resolution for $0.662 \mathrm{MeV} \gamma$-ray from a ${ }^{137} \mathrm{Cs}$ source is about $8 \%$ to $9 \%$ for the LSO and LYSO cubic samples, and is $10 \%$ for the BGO cubic sample. This resolution is compatible with what obtained by commercially available pixel crystals of much smaller size. The corresponding resolution 


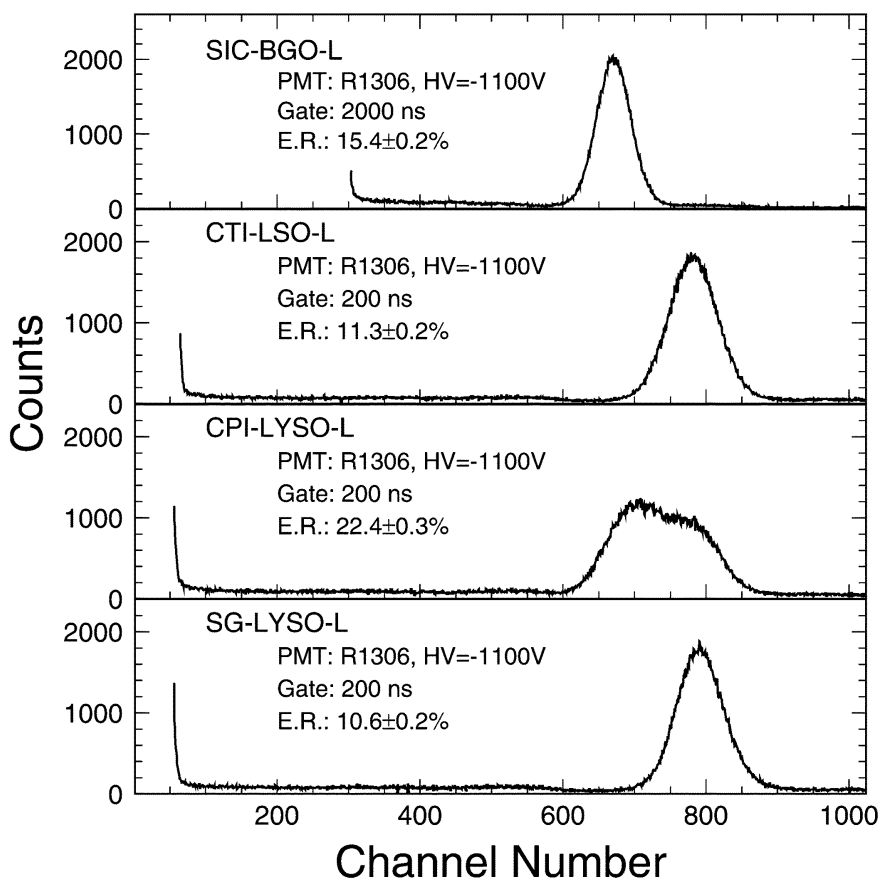

Fig. 7. The spectra of $0.51 \mathrm{MeV} \gamma$-rays from a ${ }^{22} \mathrm{Na}$ source with coincidence trigger measured by using the R1306 PMT are shown for four long samples.

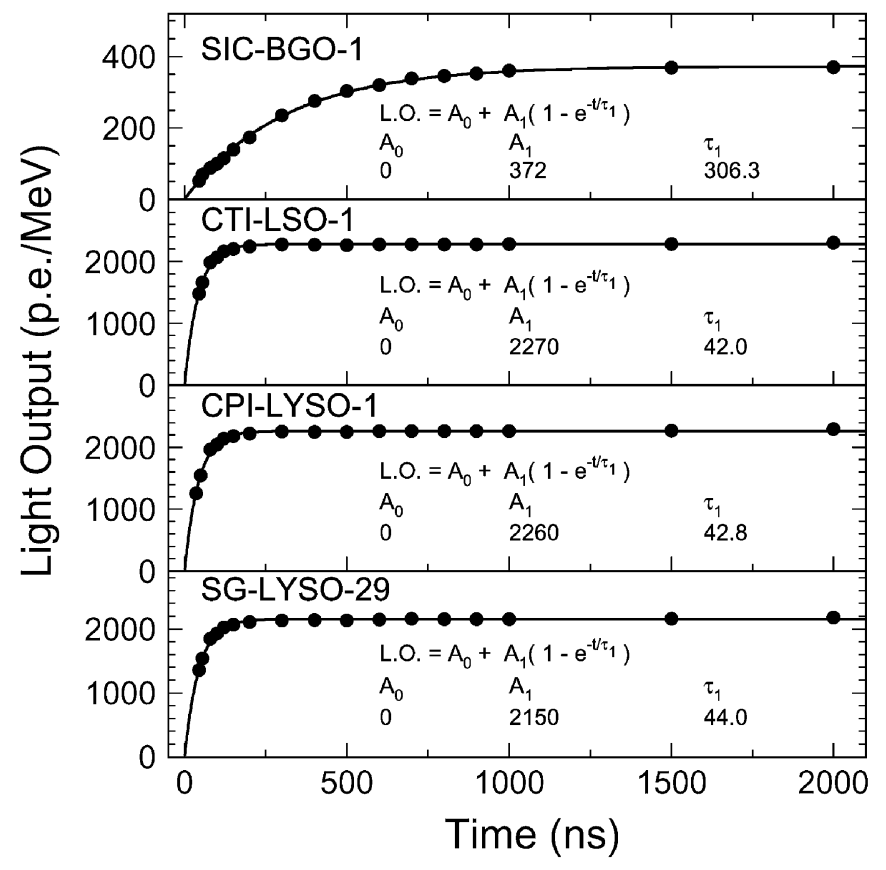

Fig. 8. Light output measured by using the XP2254b PMT is shown as a function of integration time for four cubic samples.

measured by using $0.511 \mathrm{MeV} \gamma$-rays from a ${ }^{22} \mathrm{Na}$ source with a coincidence trigger is about $10 \%$ to $11 \%$ for Saint-Gobain LYSO and CTI LSO long samples, and is $15 \%$ for the long SIC BGO sample. The CPI LYSO sample shows a double peak because of inappropriate after growth thermal annealing [8].

Figs. 8 and 9 show light output and decay kinetics observed for the cubic and long samples respectively, using the Photonis XP2254b PMT. One notes that the LSO and LYSO samples have consistent decay time and photoelectron yield. While BGO's

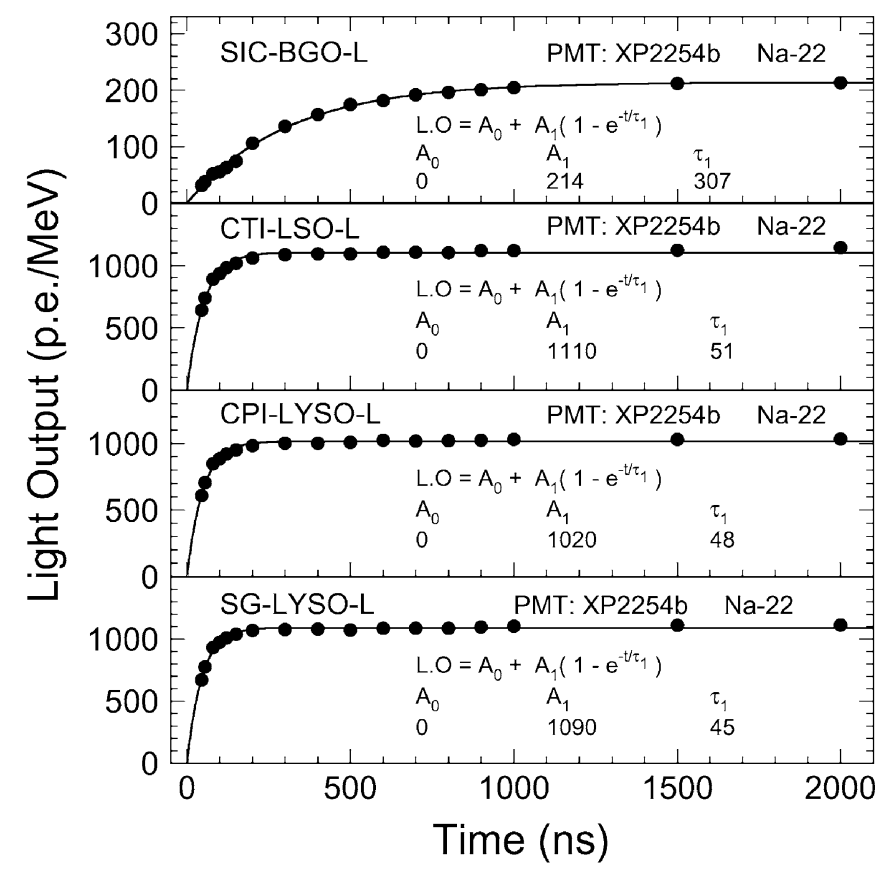

Fig. 9. Light output measured by using the XP2254b PMT is shown as a function of integration time for four long samples.

300 ns decay time is a factor of 7 slower than LSO and LYSO its photoelectron yield is a factor of six lower. Taking out the PMT response. we conclude that the light yield of LSO and LYSO crystals is a factor of four of that of BGO.

The light response uniformity of long samples was measure by allowing a collimated beam of $\gamma$-ray to aim at seven evenly distributed locations along crystal's longitudinal axis. The light output responses at these seven points were fit to a linear function,

$$
\frac{\mathrm{LO}}{\mathrm{LO}_{\text {mid }}}=1+\delta \cdot\left(x / x_{\text {mid }}-1\right)
$$

where $\mathrm{LO}_{\text {mid }}$ represents the light output at the middle of the sample, $\delta$ represents the deviation of the light response uniformity, and $x$ is the distance from the end coupled to the readout device. Because these samples have a rectangular shape, there are two ways to couple it to the PMT. The A end is defined to yield a lower average light $\left(\mathrm{LO}_{\text {mid }}\right)$ when it is coupled to the PMT. The other end is defined as the B end.

Table II summarizes the numerical result of the fit to the (3). While a consistent $\delta$ was observed for the BGO sample when both the A and B ends coupled to the PMT, different signs of $\delta$ were observed for the LSO and LYSO samples when the end coupled to the PMT is switched. This observation indicates a slight longitudinal non-uniformity of the light yield along LSO and LYSO sample's longitudinal axis.

The origin of this difference may be attributed to their chemical nature. While BGO is an intrinsic scintillator, LSO and LYSO are cerium doped scintillators. It is well known that the light output of these crystals is affected by both the cerium concentration and the yttrium fraction [7]. Any longitudinal variation of the cerium concentration and/or the yttrium fraction in LSO/LYSO would affect long sample's light response uniformity. To achieve and maintain the excellent energy resolution 
TABLE II

RESULT OF LIGHT RESPONSE UNIFORMITY WITH PMT READOUT

\begin{tabular}{ccccc}
\hline \hline $\begin{array}{c}\text { Sample } \\
\text { ID }\end{array}$ & \multicolumn{2}{c}{ A end coupled to PMT } & \multicolumn{2}{c}{ B end coupled to PMT } \\
\hline SIC-BGO & LO $_{m i d}($ p.e./MeV $)$ & $\delta(\%)$ & LO $_{\text {mid }}$ (p.e./MeV) & $\delta(\%)$ \\
CTI-LSO & 209 & $0 \pm 1$ & 207 & $0 \pm 1$ \\
CPI-LYSO & 1,070 & $1 \pm 1$ & 1,090 & $-8 \pm 1$ \\
SG-LYSO & 950 & $1 \pm 1$ & 990 & $-5 \pm 1$ \\
\hline \hline
\end{tabular}

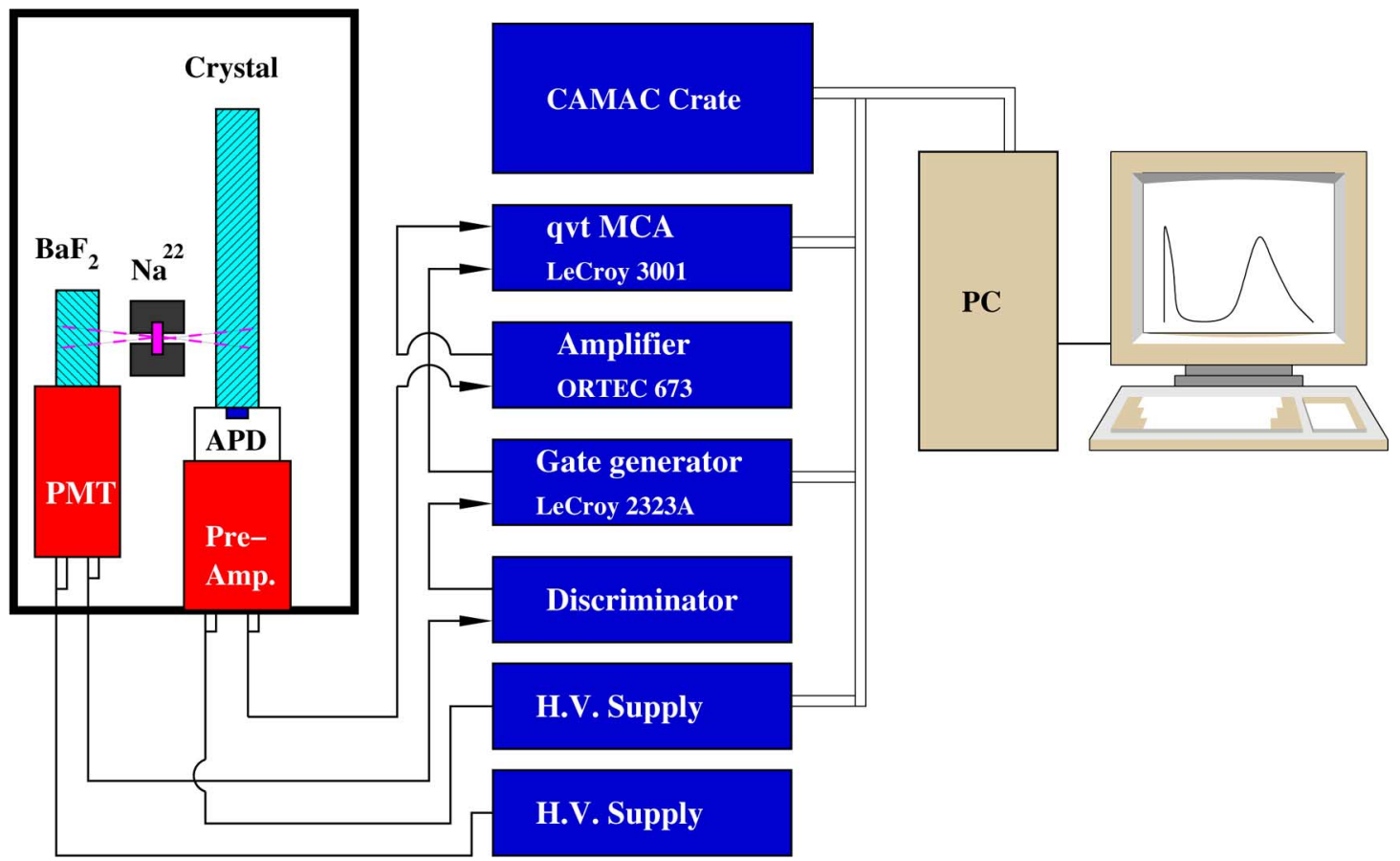

Fig. 10. A schematic showing the set-up used for the light output measurement with the APD readout and a coincidence trigger.

promised by a crystal calorimeter, effort must be made to develop long crystals of consistent light response uniformity, even in a radiation environment [12], [13].

\section{RESULT With APD READOUT}

Crystal calorimeters in high energy physics experiments are often located in a magnetic field. This excludes the use of PMT as the readout device in most cases. Solid state devices, such as silicon photodiode (PD) or silicon avalanche photodiodes (APD), are usually used since they are immune to the magnetic field. Because of lacking sufficient amplification, the APD readout would lead to a larger readout noise as compared to that of the PMT readout. For crystals with low light output, such as lead tungstate (PWO), laboratory measurements are often carried out with PMT readout. Because of their high light output, however, the light output of the LSO and LYSO samples may also be measured by using silicon APDs in laboratory.

Two commercial available Hamamatsu S8664-55 APDs were used as the readout device, which has a dimension of $5 \times 5 \mathrm{~mm}$. The total readout area was $0.5 \mathrm{~cm}^{2}$, corresponding to $17.3 \%$ and $8 \%$ coverage for the cubic and long samples respectively.
This low coverage was more or less compensated by their high (82\%) quantum efficiency. The APDs were reverse biased at $400 \mathrm{~V}$ with a gain about 50 . Their output went through a Canberra 2003 BT preamplifier and an ORTEC 673 shaping amplifier with shaping time set at 250 ns. Fig. 10 shows a schematic of the APD readout with a coincidence trigger provided by a $\mathrm{BaF}_{2}$ counter.

The top plot of Fig. 11 shows a pedestal spectrum obtained with a random trigger and a Gaussian fit with a width of 34 channels. The bottom plot of Fig. 11 shows the spectrum taken with a ${ }^{55} \mathrm{Fe} \mathrm{X}$-ray calibration, leading to 1.66 electrons per channel, where a lower gain (78\%) for the $5.9 \mathrm{keV} \mathrm{X-ray} \mathrm{from} \mathrm{a}{ }^{55} \mathrm{Fe}$ source at high reverse bias $(400 \mathrm{~V})$ was taken into account in this calibration. When the reverse bias increases the ratio between APD gains to an $\mathrm{x}$-ray source and a LED source decreases. This is due to the fact that a fraction of the X-ray energies deposited in the widened avalanche region does not receive a full multiplication. The top plot of Fig. 12 shows the ratio between the APD responses to $5.9 \mathrm{KeV}$ x-ray from a ${ }^{55} \mathrm{Fe}$ source $\left(\mathrm{R}_{\mathrm{X} \text {-ray }}\right)$ and to a blue LED light $\left(\mathrm{R}_{\mathrm{LED}}\right)$ as a function of the reverse bias voltage. $78 \%$ gain corrections were determined for the $5.9 \mathrm{KeV}$ X-ray with the reverse bias at $400 \mathrm{~V}$. The bottom plot of Fig. 12 


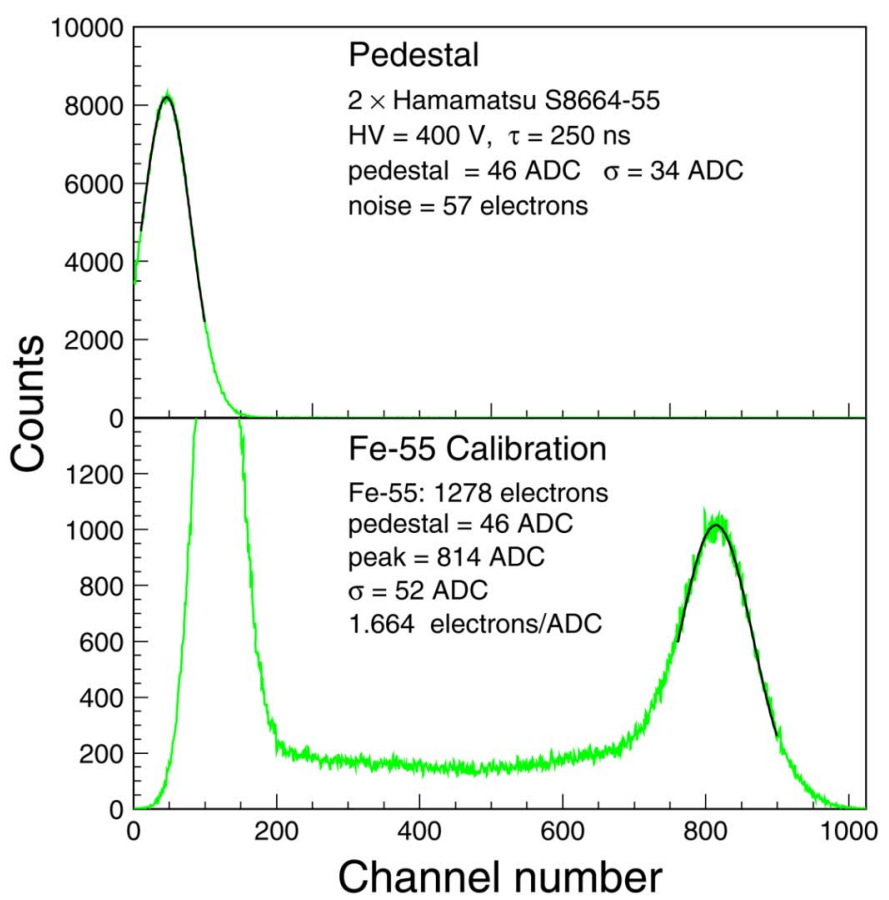

Fig. 11. Calibration of the APD readout. Top: pedestal. Bottom: ${ }^{55} \mathrm{Fe} \mathrm{X}$-ray calibration.
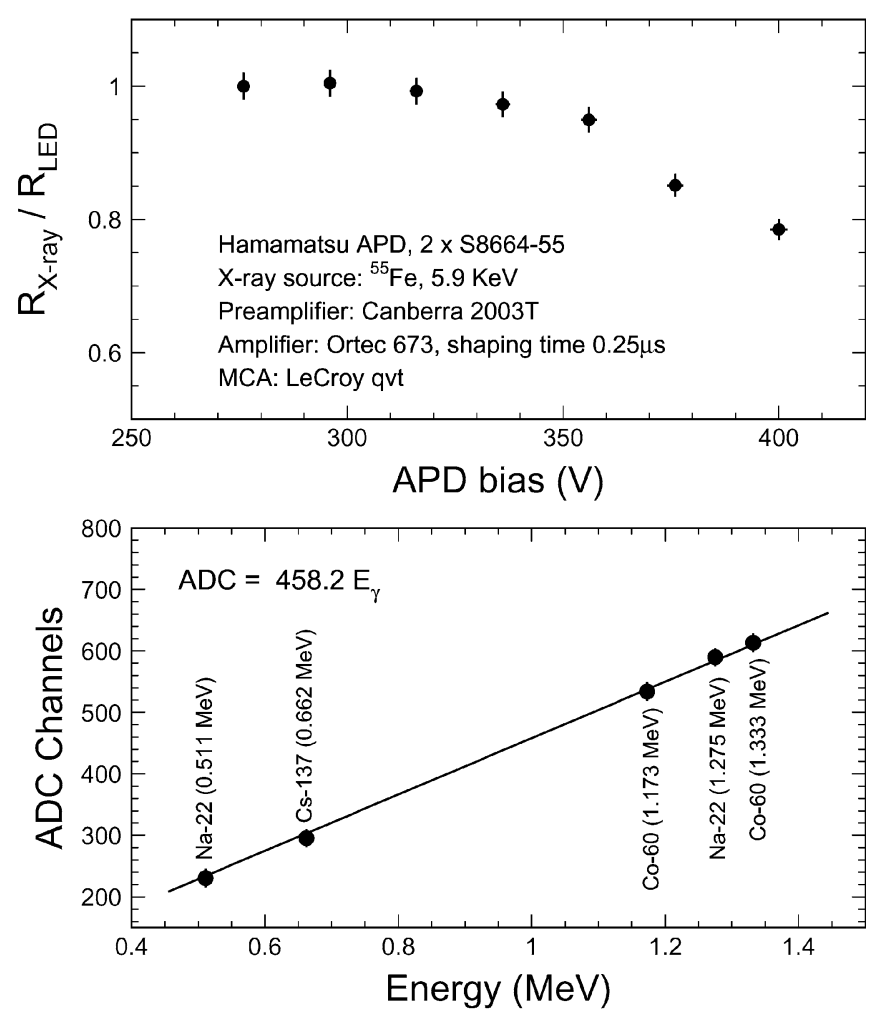

Fig. 12. Top: The ratio of APD responses to the $5.9 \mathrm{KeV}$ x-ray from a ${ }^{55} \mathrm{Fe}$ source and the blue LED light is shown as function the reverse bias voltage, indicating a lower gain with high reverse bias for the $\mathrm{x}$-ray caused by conversion in avalanche region. Bottom: A linear fit to the $\gamma$-ray peaks observed by a cubic LSO sample with the APD readout.

shows excellent linearity observed for the reconstructed peak positions determined by using various radioactive sources.

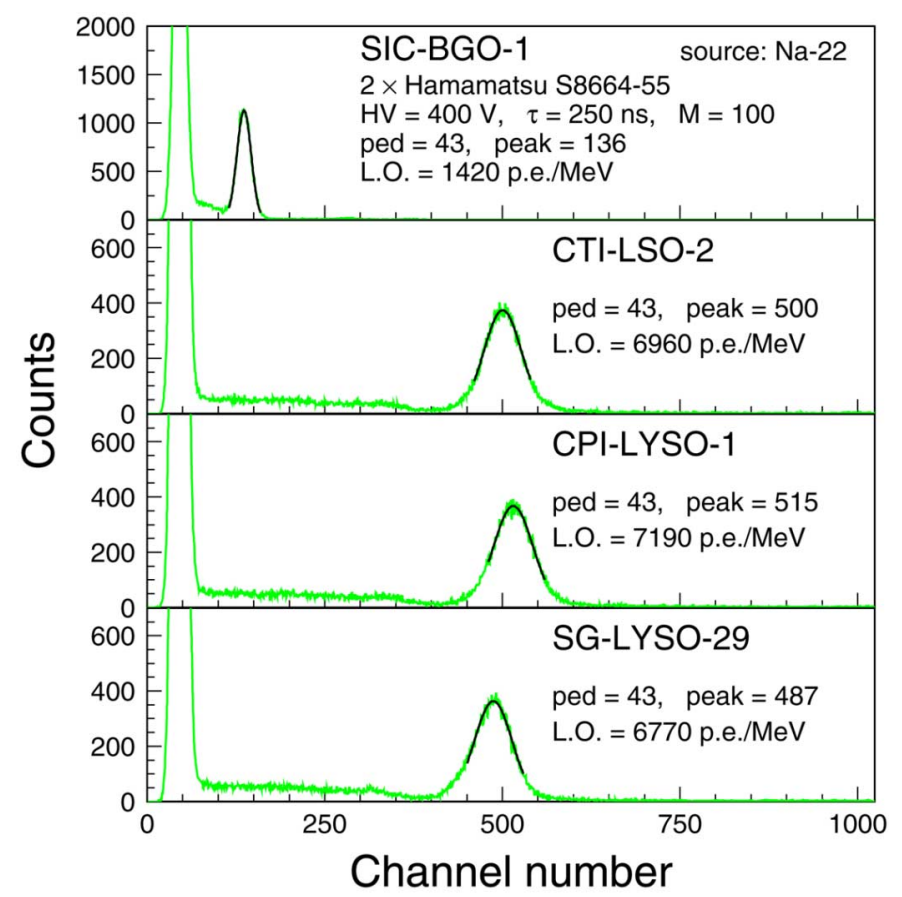

Fig. 13. The spectra of $0.511 \mathrm{MeV} \gamma$-rays from a ${ }^{22} \mathrm{Na}$ source with a coincidence trigger measured using two Hamamatsu S8664-55 APD directly coupled to the crystal are shown for four cubic samples, where $\tau$ is the shaping time and $\mathrm{M}$ is the gain setting for the ORIEL 673 amplifier.

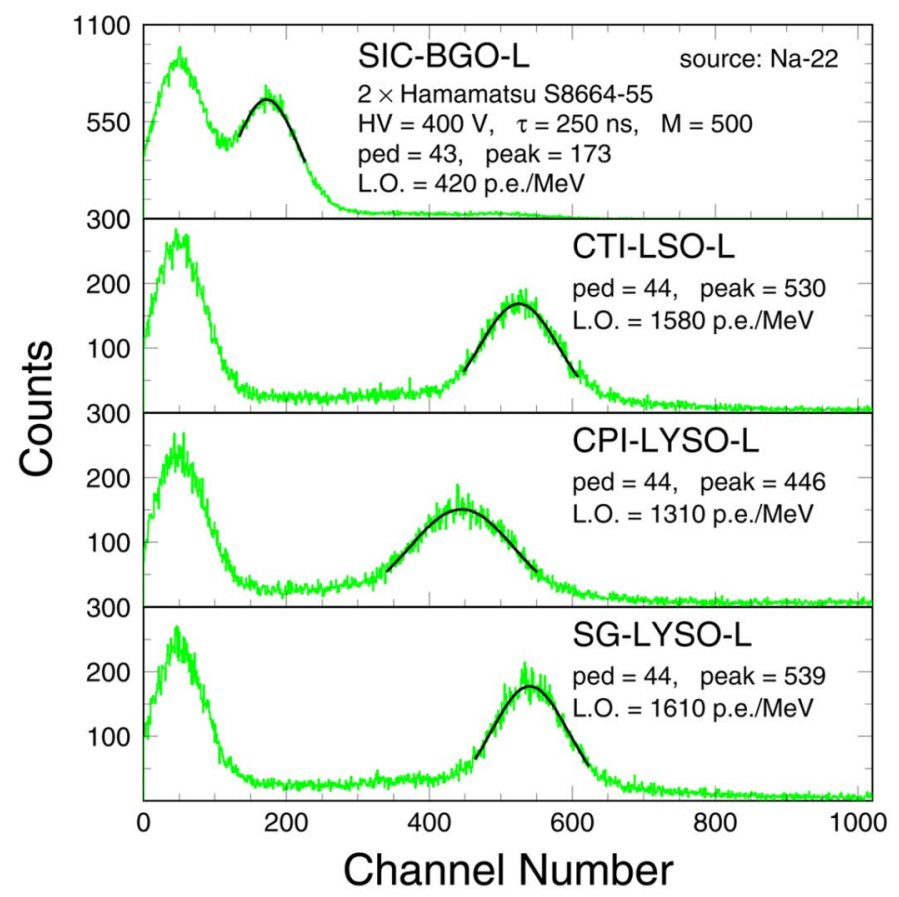

Fig. 14. The spectra of $0.511 \mathrm{MeV} \gamma$-rays from a ${ }^{22} \mathrm{Na}$ source with a coincidence trigger measured using two Hamamatsu S8664-55 APD directly coupled to the crystal are shown for four long samples, where $\tau$ is the shaping time and $\mathrm{M}$ is the gain setting for the ORIEL 673 amplifier.

This calibration was used to determine the readout noise to be 57 electrons. The stability of this readout with APDs directly coupled to the crystals was also measured to be better than $2 \%$. A better stability at $1 \%$ level by repeated measurements was achieved when the APDs are glued to a quartz plate which then 
TABLE III

RESULT OF Light RESPONSE UNIFORMITY WITH APD READOUT

\begin{tabular}{lcccc}
\hline \hline $\begin{array}{c}\text { Sample } \\
\text { ID }\end{array}$ & \multicolumn{2}{c}{ A end coupled to APDs } & \multicolumn{2}{c}{ B end coupled to APDs } \\
\hline SIC-BGO & $\mathrm{LO}_{\text {mid }}($ p.e./MeV) & $\delta(\%)$ & $\mathrm{LO}_{\text {mid }}($ p.e./MeV) & $\delta(\%)$ \\
CTI-LSO & 420 & $0 \pm 2$ & 430 & $1 \pm 2$ \\
CPI-LYSO & 1,580 & $3 \pm 2$ & 1,610 & $-7 \pm 2$ \\
SG-LYSO & 1,310 & $3 \pm 2$ & 1,320 & $-10 \pm 2$ \\
\hline \hline
\end{tabular}

coupled to the crystals through optical grease. Figs. 13 and 14 show spectra of $0.511 \mathrm{MeV} \gamma$-rays from a ${ }^{22} \mathrm{Na}$ source measured with coincidence triggers for the cubic and long samples respectively with APDs directly coupled to the crystals. The $\gamma$-ray peaks are clearly identified for all samples, although the long BGO sample shows a less distinguished peak.

The overall consistency between the light output measured by using the APD readout and that with the PMT readout is good. Assuming that the light output of long LSO/LYSO samples with the APD readout is about 1500 p.e./MeV, the energy equivalent noise can be calculated to be less than $40 \mathrm{keV}$ equivalent.

The APD readout was also used to measure light response uniformities for long LSO and LYSO samples. Table III summarizes the numerical fit result to the (3). Once again, we observed no coupling end dependence for the BGO sample. A somehow stronger coupling end dependent response, however, was observed for the long LSO and LYSO samples caused by longitudinal non-uniformity of these crystals.

\section{CONCLUSION}

Ce doped LSO and LYSO crystals have similar photo luminescence, excitation and transmittance spectra. The amplitude of their fast scintillation light of $42 \mathrm{~ns}$ decay time is about 4 times that of BGO. The light output of long $\left(2.5 \times 2.5 \times 20 \mathrm{~cm}^{3}\right)$ LSO and LYSO crystal samples, excited by $0.51 \mathrm{MeV} \gamma$-ray, can be measured by using two Hamamatsu S8864-55 APDs $\left(2 \times 0.25 \mathrm{~cm}^{2}\right)$ in laboratory with an energy equivalent noise of less than $40 \mathrm{keV}$. The absorption edge in their transmittance spectrum affects their light output. One approach to improve their light output is to shift their absorption edge toward a shorter wavelength. The light response uniformity of long LSO and LYSO samples, however, is suspected to be affected by the variation of the Ce concentration along crystal's axis.
With its intrinsic high density and high light yield of fast decay time, LSO and LYSO crystals are a good candidate for future crystal calorimeter in high energy and nuclear physics experiments.

\section{ACKNOWLEDGMENT}

The authors would like to thank Dr. B. Chai, Dr. C. Melcher, and Dr. D. Rothan for their many useful discussions.

\section{REFERENCES}

[1] K. Takagi and T. Fakazawa, "Cerium activated $\mathrm{Gd}_{2} \mathrm{SiO}_{5}$ single crystal scintillator," Appl. Phys. Lett., vol. 42, pp. 43-45, 1983.

[2] C. Melcher and J. Schweitzer, "Cerium-doped lutetium oxyorthosilicate: A fast, efficient new scintillator," IEEE Trans. Nucl. Sci., vol. 39, no. 4, pp. 502-505, Aug. 1992.

[3] D. W. Cooke, K. J. McClellan, B. L. Bennett, J. M. Roper, M. T. Whittaker, and R. E. Muenchausen, "Crystal growth and optical characterization of cerium-doped $\mathrm{Lu}_{1.8} \mathrm{Y}_{0.2} \mathrm{SiO}_{5}$," J. Appl. Phys., vol. 88, pp. 7360-7362, 2000.

[4] T. Kimble, M. Chou, and B. H. T. Chai, "Scintillation properties of LYSO crystals," in Proc. IEEE Nuclear Science Symp. Conf., Nov. 2002, vol. 3, pp. 1434-1437.

[5] W. Wisniewski, "Consideration for calorimetry at a super B factory," in Proceedings of Tenth International Conference on Calorimetry in Particle Physics, R.-Y. Zhu, Ed. Singapore: World Scientific, 2002.

[6] R.-Y. Zhu, "Precision a LSO/LYSO crystal calorimeter for the ILC," in Proc. 2005 Int. Linear Collider Physics and Detector Workshop and 2nd ILC Accelerator Workshop, Snowmass, CO, ECONF C0508141:ALCPG0705, 2005.

[7] J. M. Chen, L. Y. Zhang, and R. Y. Zhu, "Large size LYSO crystals for future high energy physics experiments," IEEE Trans. Nucl. Sci., vol. 52, no. 6, pp. 3133-3140, Dec. 2005.

[8] B. Chai, private communication.

[9] D. Rothan, private communication.

[10] C. Melcher, private communication.

[11] D. A. Ma and R. Y. Zhu, "Light attenuation length of barium fluoride crystals," Nucl. Instrum. Methods Phys. Res. A, vol. A333, pp. 422-424, 1993.

[12] R.-Y. Zhu, "Radiation damage in scintillating crystals," Nucl. Instrum. Methods Phys. Res. A, vol. A413, pp. 297-311, 1998.

[13] J. M. Chen, R. H. Mao, L. Y. Zhang, and R.-Y. Zhu, "Radiation damage in large size LSO and LYSO crystal samples," in Proc. IEEE Nuclear Science Symp. Conf., San Diego, CA, Oct. 2006, vol. 2, pp. 1112-1117. 